\title{
Fuzzy Almost Generalized E-Continuous Mappings in Smooth Topological Spaces
}

\author{
B. Vijayalakshmi, A. Vadivel
}

Abstract: We introduce and study several interesting properties of fuzzy almost generalized $e_{\text {-continuous mappings }}$ in smooth topological spaces with counter examples. We also $f T_{1} e$

introduce fuzzy $r_{-} \overline{2}^{\frac{1}{2}}$-space, $r_{-f u z z y} g e_{\text {-space, }} r_{-f u z z y}$ regular ${ }^{g} e_{\text {-space and }} r$-fuzzy generalized $e_{\text {-compact space. }}$ It is seen that a fuzzy almost generalized $e$-continuous $f T_{1} e$

mapping, between a fuzzy $r-\overline{2}^{-}$-space and a fuzzy topological space, becomes fuzzy almost continuous mapping.

Index Terms: fage -continuous, $r-f g e$-space,
$r_{-} f g e_{\text {-regular space, },} r_{-} T_{\frac{1}{2} e}$-space.

\section{INTRODUCTION}

See all undefined notions, of fuzzy sets in [28], of fuzzy topolological spaces in [[1]-[4]] [[10]-[15]], [[19]-[24]] and

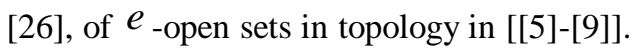

Definition 1.1 Let $f:(X, \tau) \rightarrow(Y, \sigma)$ be a mapping from a fts $\left(X, \tau_{1}\right)$ to another $\left(Y, \tau_{2}\right)$. Then $f$ is called fuzzy

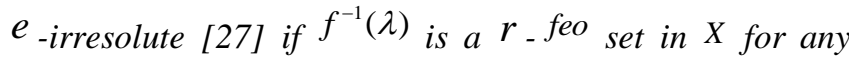
$r_{-}$feo set $\lambda$ in $Y$.

\section{FUZZY ALMOST GENERALIZED $e_{\text {-CONTINUOUS MAPPINGS }}$}

Definition 2.1 A mapping $f:(X, \tau) \rightarrow(Y, \eta)$ is said to be fuzzy almost generalized $e$-continuous (in short, fage-Cts)mapping if $f^{-1}(\lambda)$ is $r$-fuzzy generalized $e$-open (in short, $r$-fgeo) set in $X$ for every $r$-fuzzy regular open (in short, $r$-fro) set $\lambda$ in $Y$.

Theorem 2.1 Let $f$ be fage-Cts mapping iff $f^{-1}(\lambda)$ is $r$-fuzzy generalized $e$-closed (in short, $r$-fgec) set in $X$ for every $r$-fuzzy regular closed (in short, $r$-frc) set $\lambda$ in $Y$.

Revised Manuscript Received on July 08, 2019.

B. Vijayalakshmi, Assistant Professor, Dept. of Mathematics, Govt. Arts College, C. Mutlur, Chidambaram - 608102.

A. Vadivel, Assistant Professor, Dept. of Mathematics, Govt. Arts College (Autonomous), Karur - 639005 .

Example 2.1 Consider the identity mapping $f:(X, \tau) \rightarrow(X, \eta)$, where $X=\{x, y, z\}$ and fuzzy topologies (in short, FT) $\tau, \eta: I^{X} \rightarrow I$ on $\mathrm{X}$, given by

$$
\tau(\lambda)= \begin{cases}1, & \text { if } \lambda=\overline{0} \text { or } \overline{1}, \\
\frac{1}{2}, & \text { if } \lambda=\lambda_{1}, \lambda_{2}, \lambda_{3}, \lambda_{4}, \quad \eta(\lambda)=\left\{\begin{array}{ll}
1, & \text { if } \lambda=\overline{0} \text { or } \overline{1}, \\
\frac{1}{2}, & \text { if } \lambda=\lambda_{4}, \\
0, & \text { otherwise. }
\end{array}\right. \text { otherwise. }\end{cases}
$$

with

$\lambda_{1}(x)=0.4, \quad \lambda_{1}(y)=0.6, \quad \lambda_{1}(z)=0.5$

$\lambda_{2}(x)=0.6, \quad \lambda_{2}(y)=0.4, \quad \lambda_{2}(z)=0.4 ;$

$\lambda_{3}(x)=0.6, \quad \lambda_{3}(y)=0.6, \quad \lambda_{3}(z)=0.5$;

$\lambda_{4}(x)=0.4, \quad \lambda_{4}(y)=0.4, \quad \lambda_{4}(z)=0.4 \cdot$

Then $f$ is a fage-Cts mapping.

Remark 2.1 It follows from Definitions, that every fuzzy generalized e continuous (in short, fge-Cts) mapping is an fage-Cts mapping.

The forthcoming example shows that the converse of the Remark 2.1 is not true.

Example 2.2 Consider the identity mapping $f:(X, \tau) \rightarrow(X, \eta)$, where $X=\{x, y, z\}$ and FT $\tau$, $\eta: I^{X} \rightarrow I$ on $X$ given by

$$
\tau(\lambda)= \begin{cases}1, & \text { if } \lambda=\overline{0} \text { or } \overline{1}, \\
\frac{1}{2}, & \text { if } \lambda=\delta_{1}, \delta_{2}, \quad \eta(\lambda)=\left\{\begin{array}{ll}
1, & \text { if } \lambda=\overline{0} \text { or } \overline{1}, \\
\frac{1}{2}, & \text { if } \lambda=\delta_{3}, \delta_{4}, \\
0, & \text { otherwise, }
\end{array}\right. \text { otherwise. }\end{cases}
$$

with

$\delta_{1}(x)=0.3, \quad \delta_{1}(y)=0.4, \quad \delta_{1}(z)=0.5$;

$\delta_{2}(x)=0.6, \delta_{2}(y)=0.5, \quad \delta_{2}(z)=0.5$

$\delta_{3}(x)=0.4, \delta_{3}(y)=0.4, \quad \delta_{3}(z)=0.4$;

$\delta_{4}(x)=0.5, \quad \delta_{4}(y)=0.4, \quad \delta_{4}(z)=0.5$.

Then $f$ is fage-Cts mapping but $f$ is not fge-Cts mapping. Since $\omega$ is $\frac{1}{2}$-fuzzy open set in Y,but $f^{-1}(\omega)$ is not $\frac{1}{2}-$ fgeo in $X$. 
Theorem 2.2 The statements (i) - (iv) are equivalent for every fuzzy mapping $f:(X, \tau) \rightarrow(Y, \eta)$.

(1) $f$ is faeg Cts.

(2) for each fuzzy point $x_{p}$ in $X$ and each $r$-fro $q$-nbd $\delta_{2}$ of $f\left(x_{p}\right), \exists f\left(x_{p}\right) \quad q$-nbd $\lambda$ of $x_{p} \ni f(\lambda, r) \leq \delta_{2}$.
(3) $\forall \lambda$ in $X, f\left(g e C_{\tau}(\lambda, r)\right) \leq e C_{\tau}(f(\lambda), r)$.
(4) $\forall \delta_{2}$ in $Y, g e C_{\eta}\left(f^{-1}\left(\delta_{2}\right), r\right) \leq f^{-1}\left(e C_{\eta}\left(\delta_{2}, r\right)\right)$.

Proof. $(1) \Rightarrow(2)$ : We know that $f\left(x_{p}\right)$ is a fuzzy point in $Y \forall x_{p} \in I^{X}$. Then Now, let $\delta_{2} \in I^{Y}$ be a $r$-fro э $f\left(x_{p}\right) q \delta_{2}$. For, $\lambda=f^{-1}\left(\delta_{2}\right)$ since $f$ is fage continuous and $\delta_{2}$ is $r$-fro set in $X$, we have $\lambda$ is $r$-fgeo set of $X$ and $x_{p} \in \lambda$. Therefore, $\left.f(\lambda, r)=f\left(f^{-1}\left(\delta_{2}\right), r\right)\right) \leq \delta_{2}$.

$(2) \Rightarrow(3)$ : Let $x_{p} \in g e C_{\tau}(\lambda, r)$. Then $x_{p} q \lambda$

and $f\left(x_{p}\right) q f(\lambda)$ implies $\left.f\left(x_{p}\right) \in e C_{\tau}(f(\lambda), r)\right)$ and $x_{p} \in f^{-1}\left(e C_{\tau}(f(\lambda), r)\right)$.

Therefore,

$g e C_{\tau}(\lambda, r) \leq f^{-1}\left(e C_{\tau}(f(\lambda), r)\right.$.

$(3) \Rightarrow(4)$ and $(4) \Rightarrow(1)$ are clear.

Theorem 2.3 A fage-Cts mapping $f:\left(X, \tau_{1}\right) \rightarrow\left(Y, \tau_{2}\right)$ with $\left(X, \tau_{1}\right)$ be an $r$-fuzzy $T_{\frac{1}{2}} e$ (in short, $f T_{\frac{1}{2}} e$ )-space is fuzzy almost continuous (in short, fa-Cts).

Proof. Let $\lambda \in I^{Y}$ be any $r$-frc. As $f$ is fage-Cts, $f^{-1}(\lambda) \in I^{X}$ is $r$-fgec and since $X$ is $r$-fT $e$-space,

$f^{-1}(\lambda)$ is $r$-fuzzy closed (in short, $r$-fc) set in $X$. Thus $f$ is fa-Cts.

Example 2.3 Consider the identity mapping $f:(X, \tau) \rightarrow(X, \eta)$, where $X=\{x, y, z\}$ and FT $\tau$, $\eta: I^{X} \rightarrow I$ on $\mathrm{X}$, given by

$$
\tau(\lambda)= \begin{cases}1, & \text { if } \lambda=\overline{0} \text { or } \overline{1}, \\
\frac{1}{2}, & \text { if } \lambda=\delta_{1}, \delta_{2}, \delta_{3}, \delta_{4}, \quad \eta(\lambda)=\left\{\begin{array}{ll}
1, & \text { if } \lambda=\overline{0} \text { or } \overline{1}, \\
\frac{1}{2}, & \text { if } \lambda=\delta_{5}, \\
0, & \text { otherwise. }
\end{array}\right. \text { otherwise. }\end{cases}
$$

with

$\delta_{1}(x)=0.3, \delta_{1}(y)=0.4, \delta_{1}(z)=0.5$;

$\delta_{2}(x)=0.6, \delta_{2}(y)=0.5, \delta_{2}(z)=0.5$;

$\delta_{3}(x)=0.6, \delta_{3}(y)=0.5, \delta_{3}(z)=0.4$;

$\delta_{4}(x)=0.3, \delta_{4}(y)=0.4, \delta_{4}(z)=0.4$;

$\delta_{5}(x)=0.8, \delta_{5}(y)=0.4, \delta_{5}(z)=0.5$.

Then $f$ is a fuzzy generalized $e$-irresolute (in short, fge -Irr.) and fuzzy $e$-irresolute (in short, fe -Irr.) mapping.
Theorem 2.4 If $f: X \rightarrow Y$ is fge-Irr. and $g: Y \rightarrow Z$ is fage-Cts, then $g$ of $: X \rightarrow Z$ is fage-Cts.

Proof. Let $\lambda \in I^{Z}$ be an $r$-fro. Since $g$ is fage-Cts, $g^{-1}(\lambda) \in I^{Y} \quad$ is $\quad r$-fgeo. As $f$ is fge-Irr., $f^{-1}\left(g^{-1}(\lambda)\right) \in I^{X} \quad$ is $\quad r \quad$-fgeo. This implies $(g \circ f)^{-1}(\lambda) \in I^{X}$ is $r$-fgeo. Thus, $g \circ f$ is fage-Cts.

Theorem 2.5 If $f: X \rightarrow Y$ is an fuzzy continuous (In short, $f$-Cts) mapping and $g: Y \rightarrow Z$ is an fage-Cts mapping, where $Y$ is $r-f T_{1} e$-space, then $g$ of $: X \rightarrow Z$ is fa-Cts mapping.

Proof. Let $\lambda \in I^{Z}$ be any $r$-frc. Since $g$ is fage-Cts mapping, $\quad g^{-1}(\lambda) \in I^{Y} \quad$ is $\quad r$-fgec. As $Y$ is $r$ - $f T_{1} e$-space, $g^{-1}(\lambda) \in I^{Y}$ is $r$-fc. This implies $Y-g^{-1}(\lambda)$ is $r$-fo. As $f$ is $\mathrm{f}-\mathrm{Cts}$ $f^{-1}\left(Y-g^{-1}(\lambda)\right) \in I^{X}$ is $r$-fo $\Rightarrow X-f^{-1}\left(g^{-1}(\lambda)\right)$ is $r$-fo $\Rightarrow f^{-1}\left(g^{-1}(\lambda)\right.$ is $r$-fc $\Rightarrow(g \circ f)^{-1}(\lambda)$ is $r$-fc Hence, $g$ o $f$ is fa-Cts.

\section{FUZZY GENERALIZED $e$-COMPACT SPACE AND FUZZY REGULAR ${ }^{g e}$-SPACE}

Here we introduce the definition of fuzzy generalized $e_{\text {(in short, fge)-compact space and fuzzy regular }} g e_{\text {(in }}$ short, frge)-space. Also some interesting theorems involving fge compact space and ffrge-space are established.

Definition 3.1 Anfts $(X, \tau)$ is said to be $r$-fge-compact space if every $r$-fuzzy generalized $e$ open (in short, $r$-fgeo) cover of $X$ has a finite subcover.

Theorem 3.1 An fage Cts surjection image of a fge compact space is fuzzy nearly (in short, fn) compact.

Proof. Let $f: X \rightarrow Y$ be an fage-Cts surjection mapping and $\left\{\lambda_{i}\right\}_{i \in \Lambda}$ be an $r$-fro cover of $Y$. Since $X$ is fge compact, $\left\{f^{-1}\left(\lambda_{i}\right)\right\}_{i \in \Lambda}$ is a family of $r$-fgeo cover of $X$. So, there exists a finite subset $\Lambda_{0}$ of $\Lambda$ such that $\left\{f^{-1}\left(\lambda_{i}\right)\right\}_{i \in \Lambda_{0}}$ covers $X$. Since $f$ is 1-1, onto, $\left\{\lambda_{i}\right\}_{i \in \Lambda_{0}}$ will be finite $r$-fro subcover of the $r$-fro cover $\left\{\lambda_{i}\right\}_{i \in \Lambda}$ of $Y$. So, $Y$ is fn compact.

Definition $3.2 \quad A n \quad f t s$ 
$(X, \tau)$ is said to be $r$-fuzzy regular generalised $e_{\text {(in }}$ short, frge)-space if every $r$-fgeo set in $X$ is $r$-fro.

Remark 3.1 There are spaces which are not $r$-frge-spaces.

Example 3.1 Consider the fts, $(X, \tau)$ where $X=\{x, y, z\}$ and FT

$$
\tau(\lambda)= \begin{cases}1, & \text { if } \lambda=\overline{0} \text { or } \overline{1}, \\ \frac{1}{2}, & \text { if } \lambda=\delta_{1}, \delta_{2}, \\ 0, & \text { otherwise }\end{cases}
$$

with

$$
\begin{aligned}
& \delta_{1}(x)=0.3, \quad \delta_{1}(y)=0.4, \quad \delta_{1}(z)=0.5 \\
& \delta_{2}(x)=0.7, \quad \delta_{2}(y)=0.4, \quad \delta_{2}(z)=0.5 ; \\
& \delta_{3}(x)=0.8, \quad \delta_{3}(y)=0.5, \quad \delta_{3}(z)=0.5 \\
& \delta_{4}(x)=0.8, \quad \delta_{4}(y)=0.4, \quad \delta_{4}(z)=0.5
\end{aligned}
$$

Then the fts $(X, \tau)$ is not $r$-frge-space, since $\delta_{3}$ and $\delta_{4}$ are $r$-fgeo sets in $\$ \mathrm{X}, \$$ but not $r$-fro in $(X, \tau)$.

Definition 3.3 Anfts $(X, \tau)$ is said to be $r$-fge-space if every $r_{\text {-fgeo set in }} X$ is $r_{\text {-fo. }}$.

Remark 3.2 There are spaces which are not $r$-fge-spaces.

Example 3.2 Consider the fts, $(X, \tau)$ where $X=\{x, y, z\}$ and FT

$$
\tau(\lambda)= \begin{cases}1, & \text { if } \lambda=\overline{0} \text { or } \overline{1}, \\ \frac{1}{2}, & \text { if } \lambda=\delta_{1}, \delta_{2}, \\ 0, & \text { otherwise, }\end{cases}
$$

with

$$
\begin{aligned}
& \delta_{1}(x)=0.3, \delta_{1}(y)=0.5, \delta_{1}(z)=0.2 \\
& \delta_{2}(x)=0.4, \delta_{2}(y)=0.5, \delta_{2}(z)=0.5 \\
& \delta_{3}(x)=0.5, \delta_{3}(y)=0.7, \delta_{3}(z)=0.5 \\
& \delta_{4}(x)=0.4, \quad \delta_{4}(y)=0.6, \quad \delta_{4}(z)=0.5
\end{aligned}
$$

Then the fts $(X, \tau)$ is not $r$-fge-space, since $\delta_{3}$ and $\delta_{4}$ are $r$-fgeo but not $r$-fo in $\mathrm{X}$.

Remark 3.3 It is obvious from Definition 3.2 and 3.3 that every $r$-frge space is $r$-fge-space.

Example 3.3 Consider the fts, $(X, \tau)$ where

$$
\begin{gathered}
X=\{x, y, z\} \\
\tau(\lambda)= \begin{cases}1, & \text { if } \lambda=\overline{0} \text { or } \overline{1} \\
\frac{1}{2}, & \text { if } \lambda=\delta_{1}, \delta_{2} \\
0, & \text { otherwise }\end{cases}
\end{gathered}
$$

with

$$
\begin{aligned}
& \delta_{1}(x)=0.3, \delta_{1}(y)=0.5, \delta_{1}(z)=0.2 \\
& \delta_{2}(x)=0.5, \delta_{2}(y)=0.5, \delta_{2}(z)=0.5
\end{aligned}
$$

Then the fts $(X, \tau)$ is $r$-fge-space but not $r$-frge-space since $\lambda$ is $r$-fgeo and $r$-fo but not $r$-fro set in $(X, \tau)$.

Theorem 3.2 Let $f:(X, \tau) \rightarrow(Y, \sigma)$ be fge-Cts surjection mapping. Then $Y$ is $r$-fuzzy connected if $X$ is $r$-fuzzy connected space and $r_{\text {-frge-space. }}$

Proof. Suppose $(Y, \sigma)$ be not $r$-fuzzy connected, Then $\ni \lambda \in I^{Y}$ such that $\lambda \neq 0_{Y}, 1_{Y}$ and $\lambda$ is both $r$-fo and

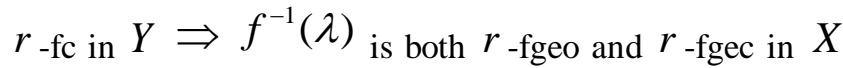
$\Rightarrow f^{-1}(\lambda)$ is both $r_{-} f r o$ and $r_{-} f r c$ in $X \Rightarrow$ $f^{-1}(\lambda)$ is both $r$-fo and $r$-fc in $X$. Also, $f^{-1}(\lambda) \neq 0_{X}, 1_{X}$. This contradicts that $X$ is a $r$-fuzzy connected space, hence, $Y$ is $r$-fuzzy connected.

Theorem 3.3 Let $f: X \rightarrow Y$ be an fage Cts surjective mapping and $X$ be $r$-frge-space as well as $r$-fn compact space. Then $Y$ is $r$-fn compact.

Proof. Let $\left\{\lambda_{i}\right\}_{i \in \Lambda}$ be any $r$-fro cover of $Y$. Since $f$ is fage Cts, $f^{-1}\left(\lambda_{i}\right)_{i \in \Lambda}$ is a family of $r$-fgeo sets in $X . X$ is $r$-frge-space. So, $f^{-1}\left(\lambda_{i}\right)_{i \in \Lambda}$ is a family of $r_{-} f r o$ sets in $X . X$ is $r$-fn compact. So, $\exists \Lambda_{0}$ of $\Lambda$ э $X \leq\left[f^{-1}\left(\lambda_{i}\right)_{i \in \Lambda_{0}}\right] . \quad$ Then $Y=f(X) \leq\left(\lambda_{i}\right)_{i \in \Lambda_{0}} \cdot$ So $\left\{\lambda_{i}\right\}_{i \in \Lambda_{0}}$ is a finite subcover of $r_{-}$fro sets of $Y$. So, $Y$ is $r$-fn compact.

Theorem 3.4 If $f: X \rightarrow Y$ is an fge Cts injective mapping and $Y$ is a $r$-fuzzy Hausorff space, then $X$ is $r$-fuzzy Hausdorff if it is $r$-frge-space. 
Proof. Let $x_{p} \neq y_{q} \in I^{X}$. Then $f\left(x_{p}\right) \neq f\left(y_{q}\right)$ in

$Y$. Since $Y$ is $r$-fuzzy Hausdorff, there exists $r$-fo nbds $U$ and $V$ of $f\left(x_{p}\right)$ and $f\left(y_{q}\right)$ respectively such that $U \wedge V=0_{X}$. Since $f$ is an fge Cts, $f^{-1}(U)$ and $f^{-1}(V)$ are $r_{\text {-fgeo in }} X \Rightarrow f^{-1}(U)$ and $f^{-1}(V)$ are $r_{-} f r o$ in $X$. [Since $X$ is $r$-frge-space] $\Rightarrow f^{-1}(U)$ and $f^{-1}(V)$ are $r$-fuzzy open in $X$ and contains respectively the fuzzy points $x_{p}$ and $y_{q}$. Now, $x_{p} \in f^{-1}(U)=\lambda, \quad$ say $y_{q} \in f^{-1}(V)=\delta_{2}$, say. So, $\lambda \wedge \delta_{2}=f^{-1}(U) \wedge f^{-1}(V)=f^{-1}(U \wedge V)=f^{-1}\left(0_{X}\right)=0_{X}$. Thus, $X$ is $r$-fuzzy Hausdorff.

\section{CONCLUSION}

It is interesting to work under a new class of mappings viz. $f T_{1} e$
fage-Cts mapping, $r-\frac{\frac{1}{2}}{\text {-space, } r \text {-fge-compact space, }}$
$r$-fge-space, $r$-frge-space in $S$ ostak's fuzzy topological spaces. It is seen that a fage-Cts surjection image of a $r$-fge-compact space is $r$-fn compact.

\section{REFERENCES}

1. K. K. Azad, On fuzzy semi continuity, fuzzy almost continuity and fuzzy weakly continuity, J. Math. Anal. Appl., 82 (1) (1981), 14-32.

2. A.S. Bin Shahna, On fuzzy strong continuity and fuzzy precontinuity, Fuzzy Sets and Systems, 44 (1991), 303-308.

3. R. N. Bhaumik and Anjan Mukherjee, Fuzzy completely continuous mappings, Fuzzy Sets and Systems, 56(2) (1993), 243-246.

4. C. L. Chang, Fuzzy topological spaces, J. Math. Anal. Appl., 24 (1968), 182-190.

5. E. Ekici, On -open sets, -sets and -sets and decompositions of continuity, Arabian Journal for Science and Engineering, 33(2A)(2008), 269-282.

6. E. Ekici, Some generalizations of almost contra-super-continuity, Filomat, 21(2) (2007), 31-44

7. E. Ekici, New forms of contra-continuity, Carpathian Journal of Mathematics, bf 24(1) (2008), 37-45.

8. E. Ekici, On -open sets and -sets, Mathematica Moravica, 13(1) (2009), 29-36.

9. E. Ekici, On -open sets -sets and decompositions of continuity and super-continuity, Annales Univ. Sci. Dudapest. Eotvos Sect. Math., 51 (2008), 39-51.

10. E. Elavarasan and A. Vadivel, -fuzzy generalized -closed sets and maps in fuzzy topological spaces, Int. J. of current Research and Modern Education., (2017), 111-115.

11. S. Ganguly and S. Saha, A note on -continuity and -connected sets in fuzzy settings, Simon Stevin, 62 (2) (1988), 127-141.

12. S. Ganguly and S. Saha, A note on compactness in fuzzy setting, Fuzzy Sets and Systems, 34(1) (1990), 117-124.

13. Y.C. Kim and J. W. Park, -fuzzy -closure and -fuzzy -closure sets, J. Korea Fuzzy Logic and Intelligent systems, 10(6) (2000), 557-563.

14. Y. C. Kim, A. A. Ramadam and S. E. Abbas, Weaker forms of continuity in ostak fuzzy topology, Indian J. Pure Appl. Math., 34(2) (2003), 311-333.

15. J. K. Kohli and A. R. Prasannan, Pseudu fuzzy continuous maps and pseudo fuzzy homeomorphism, Bull. Cal. Math. Soc., 93 (2) (2001), 69-76.
16. Mukherjee, Anjan and P. K. Deb, On fuzzy almost semi -pre continuous mappings, Proc. Nat. Sem. On Rec. Dev. In Math. And its Appl., Nov.14-15 (2008), 147-154.

17. P. Smets, The degree of belief in a fuzzy event, Information Sciences, 25(1), 1981.

18. M. Sugeno, An introductory survey of fuzzy control, Information Sciences, 36(59), 1985.

19. A. S. ostak, On a fuzzy topological structure, Rend. Circ. Matem. Palermo Ser. II 11 (1985), 89-103.

20. S. K. Samanta and K. C. Chattopadhyay, Fuzzy topology, Fuzzy closure operator, Fuzzy compactness and fuzzy connectedness, Fuzzy Sets and Systems 54 (1993), 207-212.

21. V. Seenivasan and K. Kamala, Fuzzy -continuity and fuzzy -open sets, Ann. Fuzzy Math. Inform., 8(1) (2014) 141-148.

22. V. Seenivasan and K. Kamala, Fuzzy -Compact Spaces in Fuzzy Topological Spaces, Proceedings of the International Conference on Mathematics and its Applications., (2014) 15-17.

23. D. Sobana, V. Chandrasekhar and A. Vadivel, Fuzzy -continuity in ostak's fuzzy topological spaces, (in press).

24. A. Vadivel and B. Vijayalakshmi, Mixed -fuzzy topological spaces, Int. J. of Pure and Applied Mathematics, 113(12) (2017), 115-122.

25. A. Vadivel and B. Vijayalakshmi, On fuzzy generalized -closed sets and maps in fuzzy topological spaces, (in press).

26. A. Vadivel and B. Vijayalakshmi, Fuzzy almost -continuous mappings and fuzzy -compactness, (accepted).

27. A. Vadivel and B. Vijayalakshmi, Fuzzy -irresolute mappings and fuzzy -connectedness in smooth topological spaces, (submitted)

28. L. A. Zadeh, Fuzzy sets, Information and Control, 8 (1965), 338-353

\section{AUTHORS PROFILE}

B. VijayalakshmiAssistant Professor, Dept. of Mathematics, Govt. Arts College, C. Mutlur, Chidambaram - 608102.

A. Vadivel Assistant Professor, Dept. of Mathematics, Govt. Arts College (Autonomous), Karur - 639005 . 